\title{
Highly porous activated glassy carbon film sandwich structure for electrochemical energy storage in ultracapacitor applications: Study of the porous film structure and gradient
}

\author{
Artur Braun ${ }^{\text {a) }}$ \\ Empa, Swiss Federal Laboratories for Materials Testing and Research, Laboratory for \\ High Performance Ceramics, CH-8600 Dübendorf, Switzerland; Department of Chemical and Materials \\ Engineering, University of Kentucky, Lexington, Kentucky 40506; and General Energy Research \\ Department, Paul Scherrer Institut, CH-5232 Villigen PSI, Switzerland
}

Soenke Seifert and Jan Ilavsky

Argonne National Laboratory, Advanced Photon Source, Argonne, Illinois 60439

(Received 15 December 2009; accepted 14 April 2010)

\begin{abstract}
Glassy carbon plates were thermochemically gas phase oxidized to obtain monolithic sandwichlike electrode assemblies with high surface area porous films for electrochemical energy storage applications. Film thicknesses were varied by variation of oxidation parameters time, temperature, and oxygen concentration and measured with electron microscopy. The mass density of the porous carbon film material was estimated by fitting a geometrical model to experimental gravimetric data. Optical Raman spectroscopy line scans suggest that the porosity has a gradient between the surface and the film/bulk interface, which is supported by pore-size distribution data obtained from small-angle $\mathrm{X}$-ray scattering (SAXS) on slightly oxidized and fully oxidized samples. Detailed inspection of the power law behavior of SAXS data suggests that the internal surface area of well-oxidized glassy carbon (GC) is compact and extends over the entire probed volume and thus has optimal pore connectivity. This effect goes along with pore enlargement and a relative decrease of internal surface area per volume. Slightly oxidized carbon has no pore space with a compact, high connectivity internal surface area. The corresponding SAXS power law and the x-ray density suggest that this high volumetric surface area must be interpreted as a result of surface roughness, rather than true geometric or volumetric surface area. In consequence, this surface area is of limited use for electrochemical energy storage.
\end{abstract}

\section{INTRODUCTION}

High surface area carbons are important electrode materials in electrochemical energy storage, including battery, capacitor, and fuel-cell applications. Glassy carbon (GC) is a solid and monolithic form of a hard carbon with a high number density of nanosized voids, which are not connected. ${ }^{1,2} \mathrm{GC}$ is a good diffusion barrier even to helium. Generation of high surface area in carbon is called activation. One unique property of $\mathrm{GC}$ is that it comes in monolithic form. Thin sheets make it suitable for microdevice applications. Activation of glassy carbon plates and sheets by gas phase or electrochemical oxidative treatment can be carried out in a controlled way, with theoretically well-understood kinetics and experimentally well-mastered accuracy. ${ }^{3,4}$ Oxidative treatment ${ }^{5}$ can open and connect these voids and create an active

\footnotetext{
a) Address all correspondence to this author.

e-mail: artur.braun@alumni.ethz.ch

DOI: 10.1557/JMR.2010.0197
}

carbon film with a spongelike structure and open porosity on the surface of the monolith, with internal surface areas exceeding $1000 \mathrm{~m}^{2} / \mathrm{g} .{ }^{6-10}$ Under heat in the furnace, oxygen attacks the GC surface, preferentially burns away carbon atoms which are in a fourfold atomic coordination, rather than in the threefold coordination of graphene sheets, leaving behind open and connected pores. ${ }^{7}$ These can be then exploited for technical applications. ${ }^{6,8}$ The open pores in the active film can be as small as $1 \mathrm{~nm}$ and can be filled by electrolyte ${ }^{6,7}$ and used for example for ultracapacitor electrodes. The unreacted glassy carbon core between the surface films can function as a diffusion barrier, allowing the design of bipolar electrode cell assemblies for supercapacitors with the highest power densities ever reported, see Fig. 1. With this design, performance data such as $24 \mathrm{~V} / 0.4 \mathrm{~F}$ with $10 \mathrm{~ms}$ time constant and $5.7 \mathrm{~kW}$ power are possible. ${ }^{6,11}$

During activation, the reaction front propagates normal to the GC surface into the sample interior and creates a film with open pores. Superimposed to this activation 


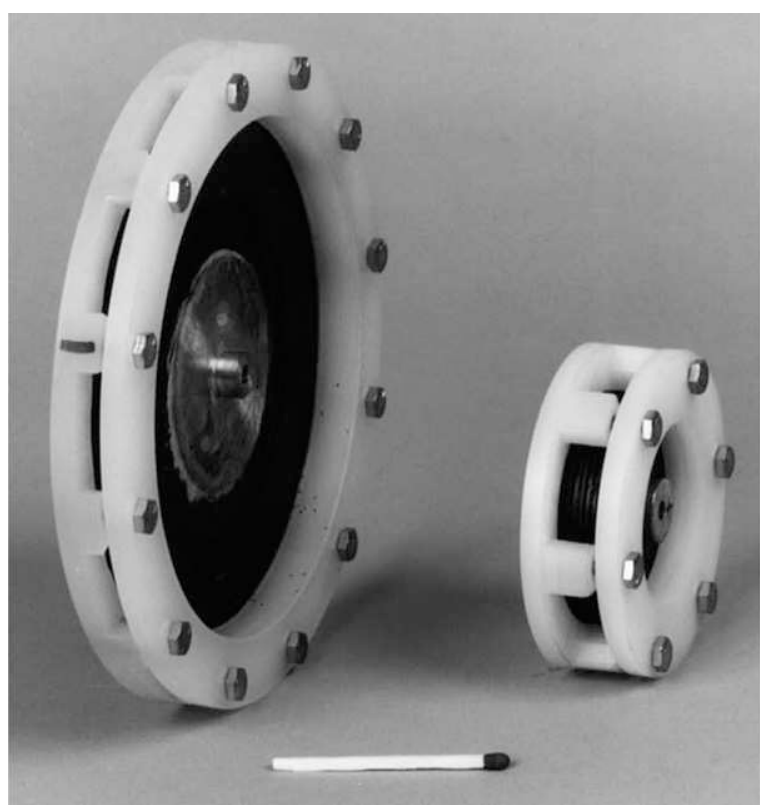

FIG. 1. GC-based supercapacitors developed at PSI Switzerland. Photo courtesy Dr. M. Bärtsch, PSI.

and film growth is the combustion, which burns off the film. ${ }^{7-10}$ Proper selection of process parameters such as temperature, oxygen concentration, and activation time, as well as the type of GC, determines how thick and fast the film will grow. ${ }^{3,4}$ The film growth problem is mathematically exactly solvable and can be written in an analytical expression, which is identical to the generalized Lambert $\mathrm{W}$ function. ${ }^{3,4}$

One of the latest trends in electrochemical energy storage and conversion is to design hybrid systems, ${ }^{12}$ which combine batteries and supercapacitors or fuel cells and batteries, for example. While the general potential of glassy carbon as supercapacitor electrode material has been specifically shown in patents, ${ }^{6}$ publications, and prototypes, ${ }^{11}$ the oxidative activation processing of glassy carbon toward an applicable electrode for electrochemical applications is a relatively complex procedure that affects the physicochemical properties at the multilength scale, starting from the surface, forming an interface and a structural gradient. Understanding of the activation process over all relevant length scales is key to optimizing the GC properties for these specific applications. We present here a novel combination of characterization techniques, which can be used to study these activated films where routine techniques (such as Brunauer-Emmett-Teller, or BET) fail.

\section{EXPERIMENTAL}

\section{A. Activation}

Glassy carbon (GC) sheets of $55 \mu \mathrm{m}$ and $1 \mathrm{~mm}$ thickness and around $1 \mathrm{~cm}^{2}$ geometrical area were thermochemically gas phase activated in an air-filled muffle furnace for times of up to $4 \mathrm{~h}$ at $450{ }^{\circ} \mathrm{C}$. For example, after $4 \mathrm{~h}$ of activation at $450{ }^{\circ} \mathrm{C}$, the entire sample thickness after activation was $21 \mu \mathrm{m}$, including $3.5 \mu \mathrm{m}$ active film thickness on both face sides. The GC was the same material as that reported in previous publications ${ }^{7-12}$ commercially named Sigradur K-type GC (Hochtemperaturwerkstoffe, Thierhaupten, Germany), pyrolyzed at $1000{ }^{\circ}$ C. Sample and film thickness were confirmed with a calibrated scanning electron microscope. Specific weight of active film and unreacted glassy carbon bulk material were obtained by a sequence of independent gravimetric and geometric measurements. Pore sizes of activated and unactivated GC were obtained with small angle x-ray scattering. ${ }^{7,13,14}$

\section{B. Ultrasmall-angle $x$-ray scattering}

Ultrasmall-angle $x$-ray scattering (USAXS) measurements were carried out at synchrotron Beamline 33-ID at UNICAT, Advanced Photon Source, Argonne National Laboratory (Argonne, IL). At this beamline, a BonseHart camera collects small-angle scattering data over a $q$ range from 0.002 to $10 \mathrm{~nm}^{-1}$ with a $q$ resolution of $0.0015 \mathrm{~nm}^{-1}$. This allows one to resolve objects with sizes from $10 \mathrm{~nm}$ to approximately $2 \mu \mathrm{m}$. Data points were distributed in approximately logarithmic scale across the $q$ range as described in Ref. 15. The X-ray energy used was $10 \mathrm{keV}$. USAXS data were fully corrected for all instrumental effects, including slit smearing. Data analysis was done with the Irena software package. ${ }^{16}$ The scattering vector $q$ of $\mathrm{x}$-rays with wavelength $\lambda$ relates to the diffraction angle $2 \theta^{17}$ via the relation $q \cdot \lambda=4 \pi \sin \theta$.

Another set of samples with $55 \mu \mathrm{m}$ nominal thickness Sigradur K activated for various times at $450{ }^{\circ} \mathrm{C}$ was measured with SAXS at HASYLAB. Small-angle X-ray scattering (SAXS) was performed at the JUSIFA Beamline of HASYLAB in Hamburg, Germany. The samples were irradiated normal to the sample surface using synchrotron radiation of $11 \mathrm{keV}$ energy. Scattering patterns were recorded using a two-dimensional position sensitive wire detector (PSD). To cover a sufficiently wide range for the scattering vector $Q$ at one particular energy, the samples were measured at a short distance $(935 \mathrm{~mm})$ and a long distance $(3636 \mathrm{~mm})$ from the detector. For calibration, a GC reference sample was measured before measuring our samples to be able to obtain absolute scattering cross-section intensities in electron units.

\section{Raman microscopy}

A confocal Raman microscope (Labram, DILOR/ Instruments Jobin Yvon S.A., Longjumeau, France) was used for the acquisition of the Raman spectra. The $530.9 \mathrm{~nm}$ line emitted by an external $\mathrm{Kr}^{+}$ion laser was used for excitation of Raman spectra in the wave number 
range of 300 to $1580 \mathrm{~cm}^{-1}$. Raman maps were obtained with a spatial resolution of $1 \mu \mathrm{m}$, using a microscope objective with $100 \times$ magnification and a pinhole diameter of $200 \mu \mathrm{m}$. To avoid changes in line shape by irradiating with high power densities, the laser power at the sample was limited to 350 to $500 \mu \mathrm{W}$. Line scans were performed across the fracture cross section with a spatial resolution of $1 \mu \mathrm{m} .{ }^{18}$ The Raman spectra were used to calculate the intensity ratio $I_{\mathrm{G}} / I_{\mathrm{D}}$ from the $\mathrm{D}$ band and $\mathrm{G}$ band at 1330 and $1575 \mathrm{~cm}^{-1}$, respectively.

\section{RESULTS AND DISCUSSION}

\section{A. Active film growth}

The left image in Fig. 2 shows the scanning electron microscopy (SEM) micrograph of the cross section of a broken thin GC sheet of around $21 \mu \mathrm{m}$ total thickness, after 210 min of activation at $450{ }^{\circ} \mathrm{C}$ in $5 \%$ oxygen. The thickness before activation was $55 \mu \mathrm{m}$. The dark region in the center (indicated as Bulk) is about $14 \mu \mathrm{m}$ thick; this is the unreacted core with closed pores and thus has no storage capacity. The brighter gray regions on the left and right (indicated as Film) show the porous active film, with a thickness of $3.5 \mu \mathrm{m}$ each.

Thermochemical gas phase activation of glassy carbon is a seemingly simple process suitable for industrial scale. The kinetics of the growth of the active film with thickness $D$ on a plate have been determined by multiple measurements of sample thickness and film thickness of number of samples grown under a wide range of conditions. The kinetics follows the generalized Lambert $\mathrm{W}$ function:

$$
D(t)=\frac{b}{a}\left\{1+\text { Lambert } W\left[-\exp \left(-1-\frac{a^{2} t}{b}\right)\right]\right\} .
$$

Constants $a$ and $b$ are the reaction rate and diffusion constant, respectively, and are temperature dependent. ${ }^{3,4}$ With a balance between reaction rate controlling the burn-off of the film and the diffusion constant controlling the film growth, the film thickness remains after some
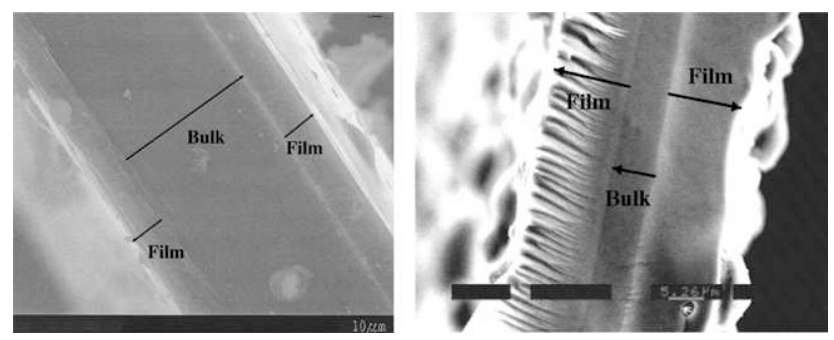

FIG. 2. Scanning electron micrographs of fracture cross sections of two activated glassy carbon samples, showing film and bulk. Image size approximately $30 \times 30 \mu \mathrm{m}$ (left, $210 \mathrm{~min}$ at $450{ }^{\circ} \mathrm{C}$ in $5 \%$ oxygen) and $60 \times 60 \mu \mathrm{m}$, respectively (right, $120 \mathrm{~min}$ at $450{ }^{\circ} \mathrm{C}$ in $20 \%$ oxygen). specific activation time constant. This steady-state film thickness is given by the ratio $b / a$, which is approached with a time constant $\tau=b / a^{2}$. Activation time essentially determines the active film thickness $D(t)$ at a given temperature and oxygen concentration. Increasing the oxidant concentration accelerates the film growth.

The evolution of the active film thickness at a given activation temperature and oxygen concentration is visualized by the sequence of SEM micrographs in Fig. 3, which were obtained from three samples activated at $450{ }^{\circ} \mathrm{C}$ in air for 60,90 , and $120 \mathrm{~min}$, with film thickness for either side being around 7, 10, and $12 \mu \mathrm{m}$, respectively.

For comparison, the right-side micrograph in Figs. 2 and 3 shows a sample from the same GC starting material, but more thoroughly activated because of higher oxygen concentration $\left(120 \mathrm{~min}\right.$ activation at $450{ }^{\circ} \mathrm{C}$ in $20 \%$ oxygen), with the total sample thickness around $30 \mu \mathrm{m}$, a total film thickness of $25 \mu \mathrm{m}$ and a nonactivated bulk thickness of $5 \mu \mathrm{m}$. To check for this value, we activated another sample for a longer time to grow a significantly thicker active film with open pores, which would consume any bulk material and coalesce at the center of the sample. This situation is realized by activating the sample for a time $t^{*}$, which can be determined by solving Eq. (1) for the activation time $t$. A GC plate with initial thickness $L$ will be totally activated after the time $t^{*}$,

$$
t^{*}=-\frac{\ln -\frac{(-2 \cdot L \cdot a-b)}{b} \cdot b+2 \cdot L \cdot a}{a^{2}} .
$$

This situation is indicated by coiling of the sample; see Fig. 4(a). The bulk material has a pronounced hardness, which gives GC its mechanical stability, owing to the sp3 bonds. When no bulk material is present anymore, this stability is lost and the material bends. Wetting one side of this fully activated GC sample causes the other side to become wet, as no gas-tight or liquid-tight barrier is left.

Figure 4(b) shows a SEM micrograph of a broken thin GC sheet of around $35 \mu \mathrm{m}$ thickness, after $1 \mathrm{~h}$ activation at $450{ }^{\circ} \mathrm{C}$. Starting thickness was $55 \mu \mathrm{m}$. The black region in the center is only about $5 \mu \mathrm{m}$ thick; this is the unreacted core with closed pores and no storage capacity. Further oxidation will further thin this core until it vanishes. The bright gray regions on the left and right show
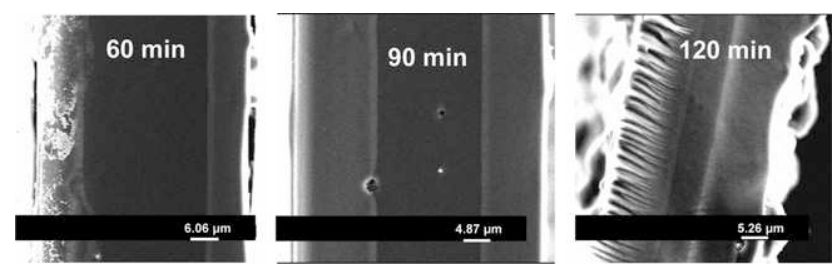

FIG. 3. Fracture planes of glassy carbon activated for 60 , 90, and $120 \mathrm{~min}$ in air in a muffle furnace, showing the relative evolution of film thickness and bulk thickness during activation. 

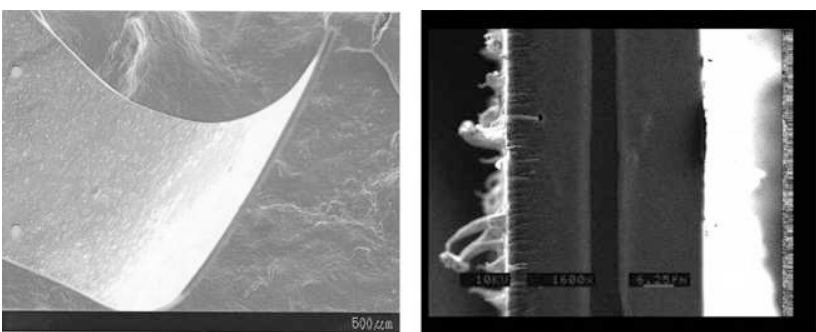

(a)

(b)

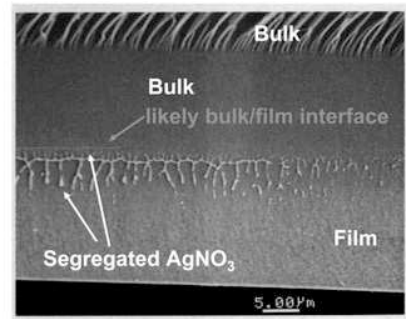

(c)

FIG. 4. Fracture cross sections of activated glassy carbon. (a) Fully activated GC with no unreacted bulk core, thus showing coiling. (b) Well-activated GC with two thick films and a thin core in between. (c) Cross section of a $1 \mathrm{~mm}$ thick activated GC sample. Bright right side shows active porous film, decorated with $\mathrm{AgNO}_{3}$ branch structure crystallized in the open pores.

the porous active film, with a thickness of $15 \mu \mathrm{m}$ each. Figure 4(c) shows $1 \mathrm{~mm}$ thick GC, with bright active film of around 20 to $30 \mu \mathrm{m}$. The activation time was $30 \mathrm{~min}$. This activated sample was soaked with aqueous $\mathrm{AgNO}_{3}$ solution to enhance the electron-optical contrast in SEM. The horizontal center line in the image shows a typical fractal pattern of segregated crystallites in the macropores of the activated GC, suggesting a preferential direction for the activation, possibly due to presence of a texture in GC.

\section{B. Optical Raman line scan profiles}

Verification for the hypothesis for an activation gradient comes by virtue of a thickness line profile analysis, which we have performed with a confocal Raman microscope. The left graph in Fig. 5 displays the intensity ratio of the $\mathrm{G}$ band versus $\mathrm{D}$ band for the nonactivated carbon as a function of the thickness coordinate. We see basically a flat, constant signal, suggesting a virtually homogeneous structure with respect to the G-band and D-band intensities. The right graph in Fig. 5 shows the intensity ratio across the partially activated glassy carbon sheet of originally (before activation) $55 \mu \mathrm{m}$ thickness. The relative intensity of the $\mathrm{G}$ band is high at the outer border, the surface of the samples. With increasing sample depth, the relative G-band intensity decreases. Across the bulk, the G-band intensity is at a low and constant plateau level similar to that of the nonactivated GC. The $\mathrm{G}$ band is indicative to graphitic structures of carbon,
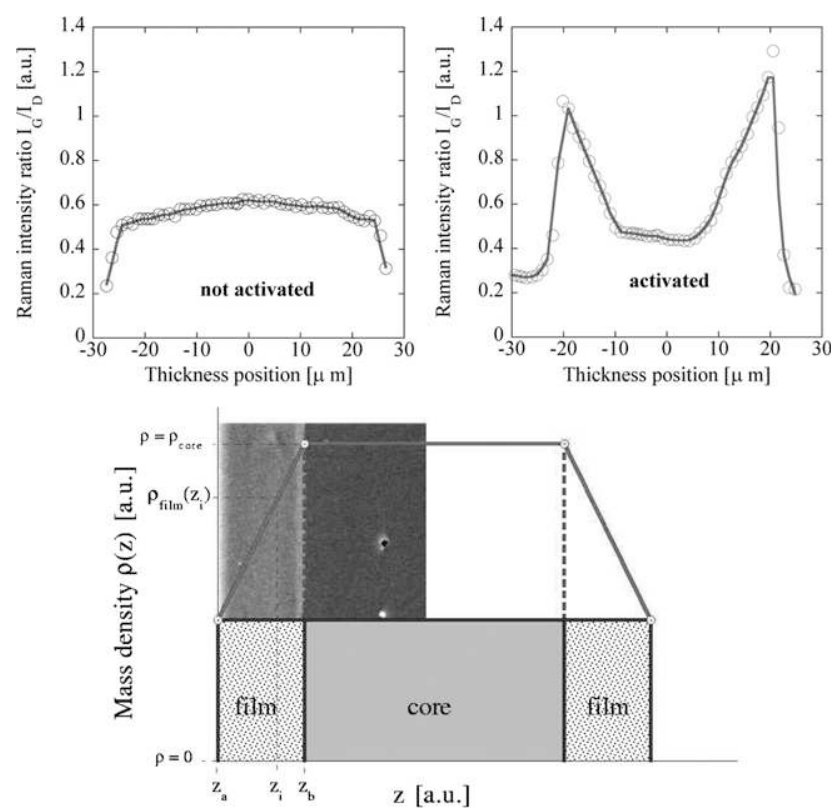

FIG. 5. Top: Two optical Raman intensity line scans across an activated GC sample cross section $\left(90 \mathrm{~min}\right.$ at $\left.450{ }^{\circ} \mathrm{C}\right)$, with underlaid SEM image for better comparison. Bottom: Schematic of mass density distribution in activated GC sheets. A GC sheet is displayed with unreacted core and active film on left and right side. A cutoff picture of a SEM micrograph is pasted over the sketch for visualization. A linear mass density gradient is assumed along the film profile. A constant mass density is assumed along the unreacted core.

suggesting that the surface of the film is more graphitic than the interior of the film and the bulk. For both samples, the decreasing intensity ratio at the sample edges may be the result of scattering artefacts. Glassy carbon is a material with mixed $\mathrm{sp}^{2}$ and $\mathrm{sp}^{3}$ carbon species. The nanosized graphite crystallites with all typical properties of graphite are bound together by carbon atoms in a tetragonal bonding, closely resembling the diamond structure, to which glassy carbon owes its pronounced mechanic hardness, and which gets lost during activation, as evidenced by the folding and bending of fully activated GC samples.

The diamondlike bonds are more prone to thermal oxidation than the graphite crystallites and are preferentially burnt off during activation. Hence, at the surface, we have a material rich in graphitic structure-as indicated by the higher relative Raman G-band intensity-while in the interior of GC we still see the form of mixed trigonal and tetragonal coordinated carbon. Because the oxidation is a diffusion-controlled process across the sample thickness, the ratio between trigonal and tetragonal carbon is a steady function of the thickness, which is reflected by the intensity variation of the G-band and D-band intensity ratio in the Raman microscopy line scan.

The density of the untreated GC is around $1.5 \mathrm{~g} / \mathrm{cm}^{3}$, depending on the type of GC (pyrolysis schedule, 
precursor). During gas phase reaction a film with open pores is created. The opening process of the pores is linked with a burn-off of material, which interlinks the graphene crystallites (tetragonal coordinated carbon, or hydrogen). The film material therefore must have a lower mass density than the original GC material with closed pores. The mass decrease caused by the thinning of the overall sample, which yields a decreasing sample thickness, can be written as a linear function of reaction time, i.e.,

$$
m(t, T)=m_{0}-a \cdot t \cdot \exp (b \cdot T) .
$$

The bottom of Fig. 5 depicts this situation with an assumed constant density across the nonactivated GC, and a decreasing density in the film toward the sample boundaries. Determination of the mass density of the active film is not trivial, because the film is bound to the unreacted core material, which has a mass density different from the film. Additionally, only small amounts of material are available for such measurements, particularly for the $55 \mu \mathrm{m}$ thin samples. Therefore, indirect methods are applied, for which $1 \mathrm{~mm}$ thick GC samples proved more convenient. The total mass of a flat monolithic GC sample with geometrical area $S_{\mathrm{p}}$ consists of the unreacted bulk (thickness $d_{\text {core }}$ ) mass and the active film (thickness $d_{\text {film }}$ ) mass:

$$
\begin{gathered}
m_{\mathrm{tot}}=\rho_{\text {core }} \cdot V_{\text {core }}+\rho_{\text {film }} \cdot V_{\text {film }}, \\
m_{\text {tot }}=S_{\mathrm{p}} \cdot \rho_{\text {core }} \cdot d_{\text {core }}+S_{\mathrm{p}} \cdot \rho_{\text {film }} \cdot 2 \cdot d_{\text {film }}, \\
m_{\text {tot }}=S_{\mathrm{p}}\left[\left(D-2 d_{\text {film }}\right) \rho_{\text {core }}+2 d_{\text {film }} \rho_{\text {film }}\right] \\
D=d_{\text {core }}+2 d_{\text {film }}
\end{gathered}
$$

where $V$ is the volume and $\rho$ is the density of each species. The overall sample thickness is $D$. The mass density $\rho_{\text {core }}$ is given in the manufacturer information and can be measured easily by its geometrical data and sample weight. The decrease of the sample thickness is directly correlated with the mass decrease and yields a linear relation: $D(t)=L_{0}-\alpha t$, where $L_{0}$ is the initial thickness of the sample and $\alpha$ is the burn-off coefficient for the sample thinning, which can be determined directly by thickness determination of samples activated for various times. The active film thickness $d_{\text {film }}$ can be approximated by a square root type law: $d_{\text {film }}(t) \approx \beta \cdot t^{1 / 2}$. From the mass changes during activation, the active film density in principal can then be derived from:

$$
\frac{m(t)}{S_{\mathrm{p}}}=\left(L_{0}-\alpha \cdot t-2 \cdot \beta \cdot \sqrt{t}\right) \cdot \rho_{\text {core }}+2 \cdot \beta \cdot \sqrt{t} \cdot \rho_{\text {film }} .
$$

Experimental data for the mass changes of Sigradur K with $1 \mathrm{~mm}$ thickness, activated at $450{ }^{\circ} \mathrm{C}$, are plotted in Fig. 6 with a fit to Eq. (8). In the plot the film-growth rate $\beta$ and the film mass density $\rho$ were independently opti-

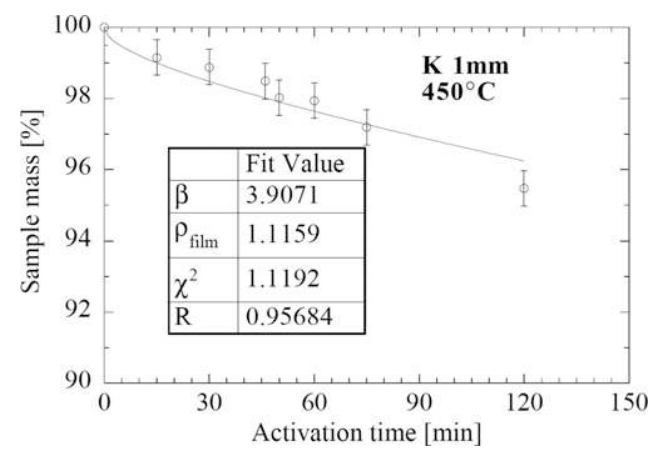

FIG. 6. Variation of sample mass from $1 \mathrm{~mm}$ thick GC with increasing activation time obtained from a set of samples activated in air at $450{ }^{\circ} \mathrm{C}$. The solid line is a least square fit to Eq. (8).

mized. In this approach, the density of the film is considered constant, although it is likely that the film density may be different at the film surface and the film/bulk interface. A film mass density of $1.1 \mathrm{~g} / \mathrm{cm}^{3}$ and a filmgrowth rate of $3.9 \mu \mathrm{m} / \mathrm{h}$ were found by fitting. A film mass density around 1.1 to $1.2 \mathrm{~g} / \mathrm{cm}^{3}$ seems rather high. However, if the mass lost during film growth was larger, this should be more pronounced in the experimental data for the weight loss. From thickness determination experiments on SIGRADUR K samples with $60 \mu \mathrm{m}$ nominal thickness a film mass density also around 1.1 to $1.2 \mathrm{~g} /$ $\mathrm{cm}^{3}$ was found. From film thickness determination and mass decrease measurements on SIGRADUR $\mathrm{K}$ with $1 \mathrm{~mm}$ thickness, a film mass density of $1.20 \mathrm{~g} / \mathrm{cm}^{3}$ was estimated by geometrical considerations.

So far we have implicitly assumed that the mass density of the active film is constant. However, the Raman spectrum profiles give evidence that the active film has a gradient in its structure, which possibly could have its origin in a mass gradient. It is not unreasonable to assume that the film near the reaction interface core/film has a larger mass than the film at the sample outer surface. Because the profile of the Raman absorbed intensity on the sample is linear, we may conclude that there is linear mass density profile between the two interfaces. The bottom part of Fig. 5 displays a schematic sketch of the distribution of the mass density versus the sample thickness line. A linear concentration gradient usually yields a square-root-like growth law for the growth of thin films. In the formulation presented previously, the film mass can be taken into account:

$$
d m_{\mathrm{film}}=\rho(z) \cdot d V(z)
$$

$$
m_{\mathrm{film}}(t)=\int d m(t)=\int_{V_{\text {film }}} \rho(z) d V(z)=S_{\mathrm{p}} \int_{z_{\mathrm{a}}}^{z_{\mathrm{b}}} \rho(z) d z
$$

The coordinate $z$ denotes the thickness changes in the sample during activation, therefore being a function of 
activation time $t: z(t)$. The integration has to be carried out between the core/film interface $z_{\mathrm{b}}$ and the outer surface $z_{\mathrm{a}}$ of the sample, both of which are functions of reaction time. Because of a lack of sufficient number of data points, the film mass density investigations did not yield an exact determination of $\rho_{\text {film }}$. The probable value should be slightly above $1 \mathrm{~g} / \mathrm{cm}^{3}$. It should be clarified in further experiments whether and how the film mass density depends on the GC structure. It would also be of fundamental interest whether the density is constant throughout the whole film or, what is expected by the author as a result of the results of the Raman experiments, the density follows a gradient that is not known yet.

Directly related with the burn-off of the tetragonal coordinated and less-ordered carbon is the evolution of the porosity in GC. We hence believe that the low G-band intensity in the bulk, increasing through the film thickness profile, qualitatively reflects the increase in porosity at the particular thickness position in the sample.

At the interface between bulk and film will be the smallest, just opened pores, because they have been exposed least to the oxidant. The pores at the outer surface of the film have been exposed most and thus have grown during oxidation. Generally, small pores allow for a larger surface area per volume than large pores. Therefore it is plausible that a GC sample that has been activated only for a short time will have smaller pores with a relatively high surface area, while after a longer time activation pores coalesce, so that the previously separating pore walls are burnt off, lowering the overall surface area. This is reflected by our observations of the dilemma that the bulk-corrected adsorption capacity of the lessactivated sample has a larger bulk-corrected storage capacity than a well-activated sample with large pores and smaller surface area. ${ }^{7}$ However, we do find that activated GC still is a high surface area active carbon, with a BET surface area close to $1000 \mathrm{~m}^{2} / \mathrm{g}$.

Similar observations on the decrease of volumetric capacity with increasing film thickness and activation were made with electrochemical experiments. The electrochemical double-layer capacity per film volume was large for samples that have been activated softly only. The total double-layer capacity per sample was increasing during activation as a result of film thickness growth, but the volumetric capacity decreased from 100 to some $90 \mathrm{~F} / \mathrm{cm}^{3}$ during activation in air from 0 to $3.5 \mathrm{~h}$ at $450{ }^{\circ} \mathrm{C}$, for instance. ${ }^{7-9}$ This behavior can be explained with pore growth and coalescence of pores, which consume part of the internal surface area during activation. While a larger volumetric surface area and related quantities, such as double-layer capacity or gas storage capacity, are generally desirable, we find that such large surface areas are restricted to particular activation parameters, such as short activation time, hence small overall capacity. In addition to that are pores so small that they present higher kinetic barriers toward mass transport, such as gas molecules or electrolyte ions, which diminish the advantage of larger capacity. For electrochemical applications, we have found that thin films with a large volumetric capacity provide an undesirable large diffusion resistance, which manifests in a large time constant. ${ }^{7}$ Because the electrochemical double-layer capacity in glassy carbon electrodes is a dispersive quantity, it depends on the frequency of the signal applied. Narrow pores limit transport. We believe that this also holds, though to a lesser extent, for gas storage applications.

\section{Small-angle $x$-ray scattering}

The GC samples that needed to be characterized for porosity pose a challenge to gas adsorption techniques. The small volume and weight of materials available makes adsorption measurements impractical. Therefore, we choose to use small-angle x-ray scattering (SAXS) to determine pore sizes of open and closed pores. It should be, however, noted that while the BET measurements are based on slit model, for analysis of SAXS data we choose to assume that the pore system is composed of spherical voids. We recognize that neither of these models is a perfect match for the real structure of porous activated GC microstructure.

Figure 7 shows SAXS curves of the two differently activated GC samples (210 and 260 min activation) and the nonactivated GC. The sample activated for $260 \mathrm{~min}$ represents a sample with no unreacted core left because of extensive activation [compare with Fig. 4(a)], while the sample with $210 \mathrm{~min}$ activation does have some unreacted core left. Figure 8 shows the same SAXS curves as Fig. 7, but after correcting the scattering contribution for the $210 \mathrm{~min}$ activated sample for bulk

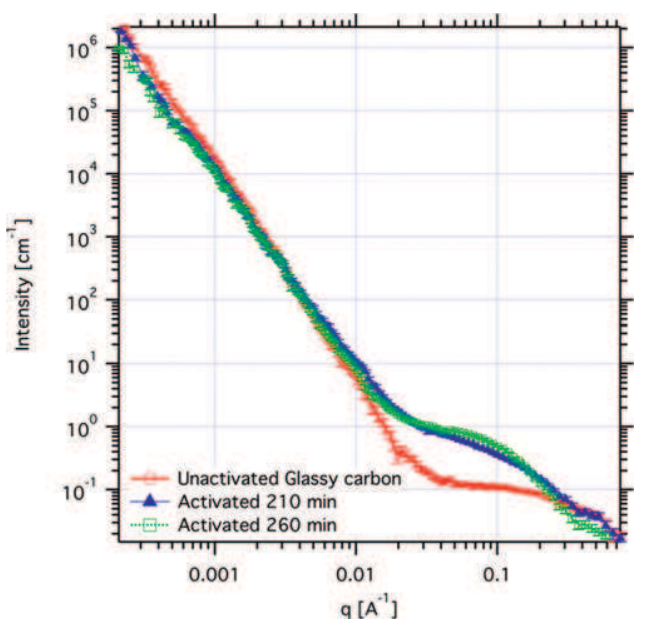

FIG. 7. SAXS curves of unactivated GC (lower SAXS curve, red), GC activated for $210 \mathrm{~min}$ (middle SAXS curve, blue) and activated for 260 min (upper curve, green). 
scattering using procedure previously published elsewhere. $^{7}$ Therefore, the two SAXS curves in Fig. 8 show only the scattering from the porous film. Micropore scattering in these SAXS curves is represented by Guinier shoulder at about $0.1 \AA^{-1}$. For the sample activated for $260 \mathrm{~min}$, this shoulder shifted to smaller $q$ values, which indicates an increase in the pore sizes.

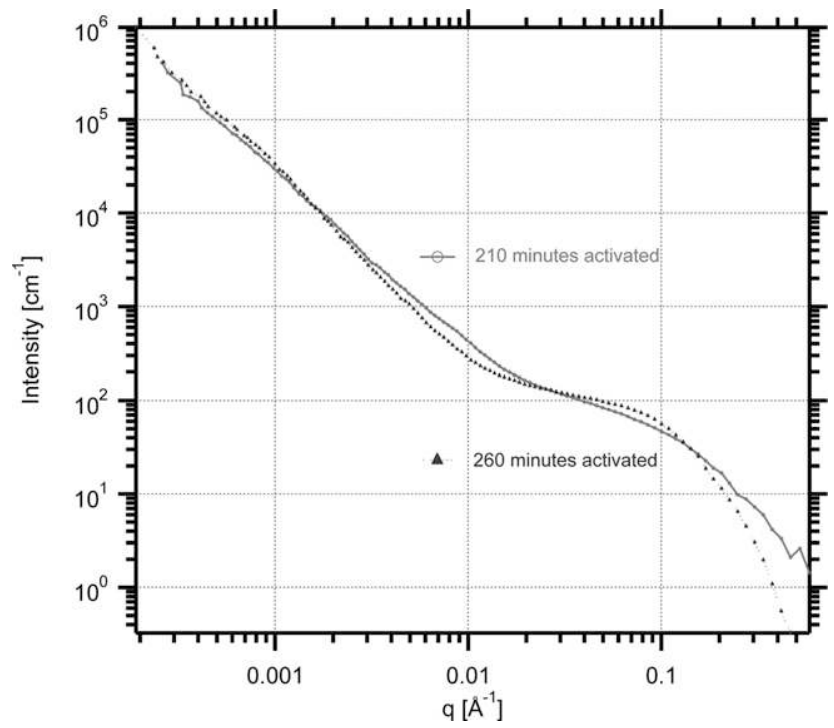

FIG. 8. SAXS curves of the activated GC samples (210 and $260 \mathrm{~min}$ ) after subtraction of the unreacted bulk scattering contribution.
Further quantitative analysis of the SAXS curves was performed using the unified fit method, ${ }^{16}$ as shown in the Fig. 9.

$$
\begin{aligned}
I(q)= & F_{\mathrm{B}}+\sum_{i=1}^{n} S_{i}(Q)\left(G_{i} \cdot \exp \left(-Q^{2} R_{\mathrm{g}_{i}}^{2} / 3\right)\right. \\
& +B_{i} \cdot \exp \left(-Q^{2} R^{2} / 3\right) \\
& \left.\times\left\{\left[\operatorname{erf}\left(Q R_{\mathrm{g}_{i}} / 6^{1 / 2}\right)\right]^{3} / Q\right\}^{P_{i}}\right)
\end{aligned}
$$

where $F_{\mathrm{B}}$ is an optional flat background, $i$ represents the structural levels, and, for each structural level $i, G_{i}$ is the exponential prefactor, $R_{g i}$ is the radius of gyration, and $B_{i}$ is a constant prefactor specific to the type of power law scattering.

Assuming dilute limit distribution of noninteracting spheres, one can analyze size distribution of pores in the GC using tools included in the Irena package. ${ }^{16}$ In this case, using the Irena modeling tool we fitted a log-normal distribution of spheres and using least square fitting optimized the distribution parameters, as well as volume of particles. As is explained below, the terminal slope of the data never reaches the Porod slope of -4 , which is common for glassy carbon materials. Therefore there are limitations on the modeling of voids using this approach-especially for the small features. With this in mind, note that Fig. 10 shows the resulting size distributions for both samples. The $210 \mathrm{~min}$ activated sample has

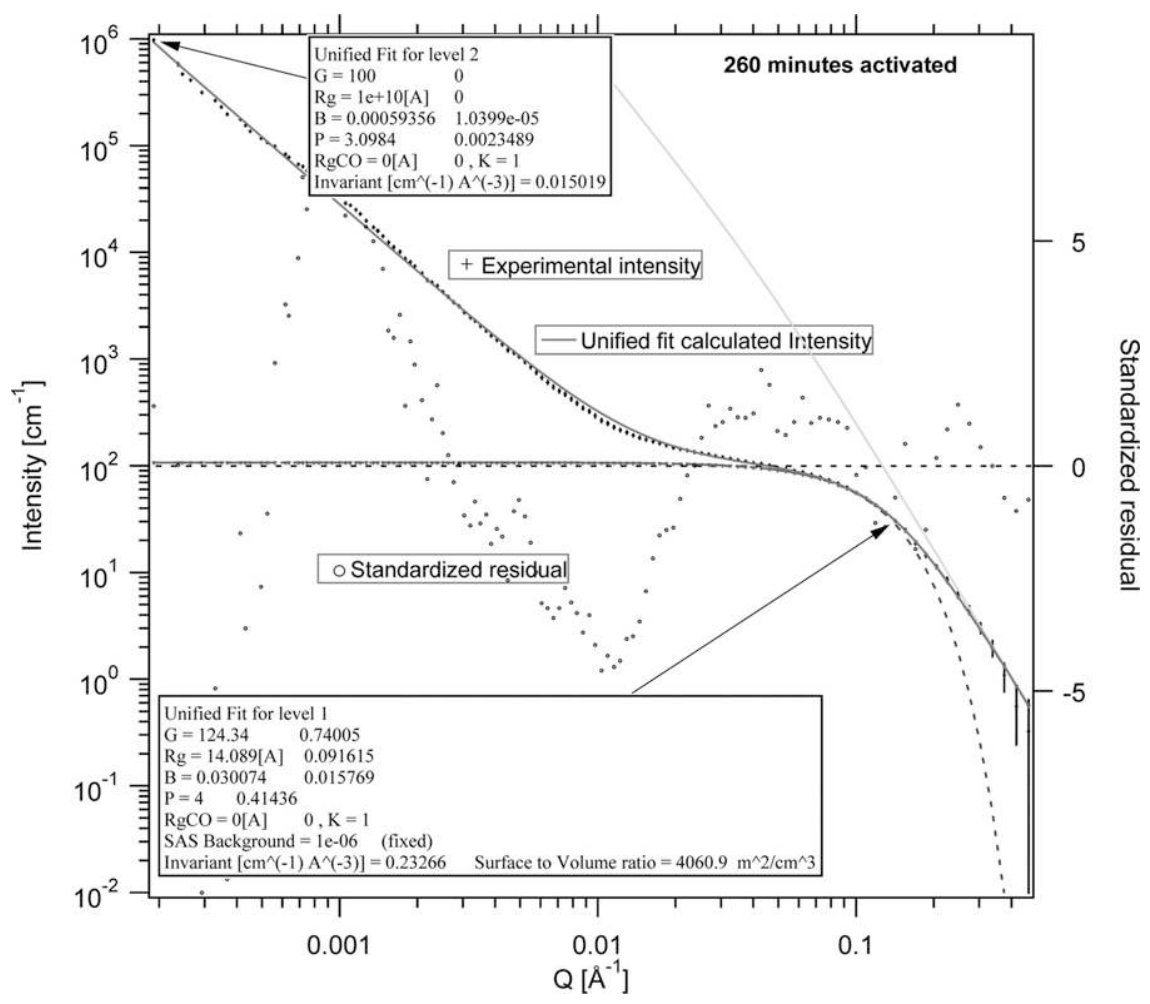

FIG. 9. USAXS curves of the 260 min activated sample, with unified fit. 


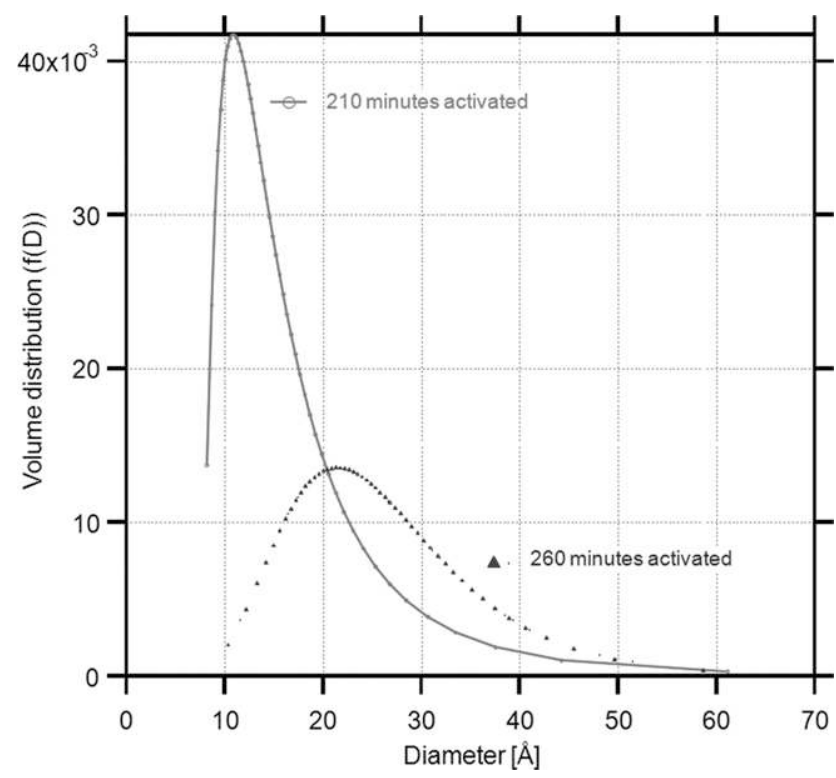

FIG. 10. Pore size for 210 and 240 min activated GC.

a maximum at $10.5 \AA$ while the 260 min activated sample has a maximum at $20.1 \AA$. Hence, on average the pores in the fully activated $(260 \mathrm{~min})$ sample are about twice as large as those in the $210 \mathrm{~min}$ activated sample.

Additional information on the structure of the GC can be inferred by analyzing the exponent of decay in the power law range of the scattering curves. The asymptotic behavior of the scattering curves for large $q$ values was studied, and we found that the slope of the scattering curves does not equal -4 , as would be expected for smooth and nonoriented voids ${ }^{17}$; for example, see Fig. 11. The slope of the large $q$-tail of many carbon materials is typically around -2.5 . Figure 11 shows a set of samples activated for different times, ranging from 1 to $165 \mathrm{~min}$. The exponent of decay is approaching -3 in the course of activation.

The indispensable feature of activated GC for electrochemical energy storage is the presence of connected pores, which can be quantified by the so-called Euler-Poincare characteristic. ${ }^{19}$ Though one of the more important, more drastic, and critical changes of the Euler-Poincaré characteristic, the number difference between connected and nonconnected pores ${ }^{19}$ has never been quantified with scattering techniques. However, subtle evidence for this transition comes from the SAXS curves. For materials with open pore surface area uniformly extended over the entire pore space, the slope must be $-3.0 .^{20}$ This is exactly the case for the SAXS curve of the $260 \mathrm{~min}$ (fully) activated GC. In the original scattering curves from nonactivated GC and activated GC (see Fig. 11), the exponent of decay of the SAXS curves for large $q$ values from -2.2 to -3.0 is a subtle indicator that the voids become connected ${ }^{20}$ and hence

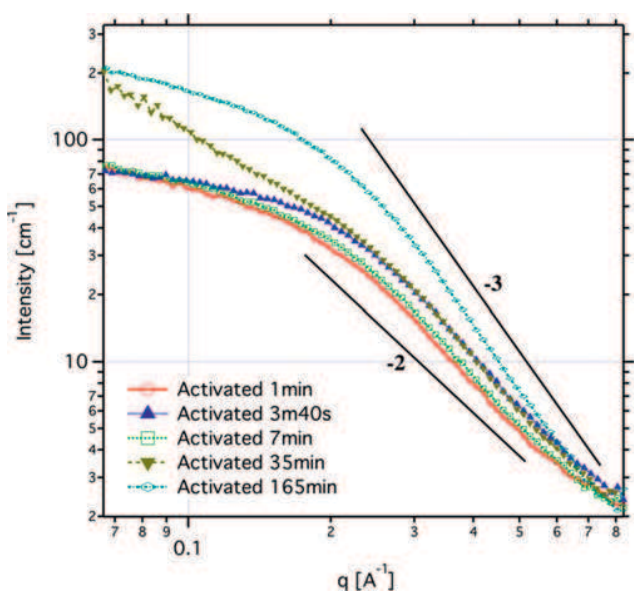

FIG. 11. SAXS curves of GC activated for $1 \mathrm{~min}, 3 \mathrm{~min} 40 \mathrm{~s}, 7 \mathrm{~min}$, $35 \mathrm{~min}$, and $165 \mathrm{~min}$, showing different exponent of decay. The exponent of decay is approaching -3 with increasing activation time.

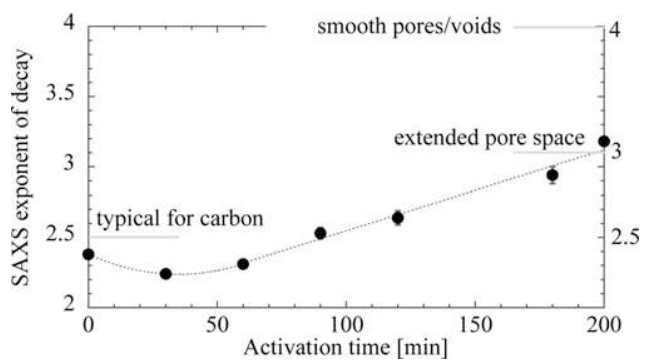

FIG. 12. SAXS exponent of decay of activated at $450{ }^{\circ} \mathrm{C}$ in air $55 \mu \mathrm{m}$ GC Sigradur K for various activation times.

become connected pores, during activation. An overview of this variation is shown in Fig. 12.

Because the activation is a continuous, gradual process, we expect that the pores in the film have a different size, depending on the depth in the film. Pores right at or near the reaction front before the bulk material are smaller than pores near the surface that have been exposed to oxygen for a longer time. Therefore, the volumetric surface area in $\mathrm{m}^{2} / \mathrm{cm}^{3}$ in a thin film would be larger than in a thick film.

It is difficult to determine the pore size gradient across the activated GC, although our USAXS experiments with bulk scattering subtraction represent a first step in this direction. More scattering experiments on a whole series of samples with different film thickness and serial subsequent subtractions would be necessary for that. A potential promising alternative could come from future USAXS instrumentation. Recently, graded solid oxide fuel cell assemblies were subject to a detailed USAXS study with a spatial resolution of $10 \mu \mathrm{m}^{21}$ With improved instrumentation, i.e., resolution at the $1 \mu \mathrm{m}$ range or better, spatial variation of the pore space in graded materials or activated GC such as presented here, could be probed with higher accuracy. 


\section{CONCLUSIONS}

The growth of activated glassy carbon films with open porosity can be visualized with electron microscopy. Crystallization of electron optical contrast enhancing salts such as $\mathrm{AgNO}_{3}$ in the porous structure shows how the oxidation front propagates in the glassy carbon pores and forms a fractal-like pore structure network and suggests an anisotropic oxidation process. Optical Raman spectromicroscopy data support the suggestion of a gradient pore structure formation during oxidation. The average density of the porous film is slightly above $1 \mathrm{~g} / \mathrm{cm}^{2}$. Extensive oxidation makes the unreacted core region in the glassy carbon vanish and causes coiling of the sample. The time and other process parameters necessary to reach this state can be derived by inversion of the generalized Lambert $\mathrm{W}$ function. Such extensively oxidized glassy carbon has a compact pore network with maximal connectivity, as was found by analysis of the power law behavior of SAXS data, i.e., a power law exponent of decay of -3 .

\section{ACKNOWLEDGMENTS}

Use of the Advanced Photon Source at Argonne National Laboratory was supported by the United States Department of Energy, Office of Science, Office of Basic Energy Sciences, under Contract No. DE-AC0206CH11357. We are grateful to Dr. J-C. Panitz (PSI) for the Raman and Dr. Yuanzhi Chen (Univ. of Kentucky) for the SEM experiments. Financial support by the European Commission (MIRG No. CT-2006-042095) is acknowledged.

\section{REFERENCES}

1. J.C. Lewis, B. Redfern, and F.C. Cowlard: Vitreous carbon as a crucible material for semiconductors. Solid State Electron. 6(3), 251 (1963).

2. G.M. Jenkins and K. Kawamura: Polymeric Carbons-Carbon Fiber, Glass and Char, 1st ed. (Cambridge University Press, Cambridge UK, 1976).

3. A. Braun, M. Bärtsch, B. Schnyder, and R. Kötz: A model for the film growth in samples with two moving boundaries-An application and extension of the unreacted-core model. Chem. Eng. Sci. 55(22), 5245 (2000).

4. A. Braun, A. Wokaun, and H-G. Hermanns: Analytical solution to a growth problem with two moving boundaries. Appl. Math. Modell. 27(1), 47 (2003).

5. J.C. Lewis, I.J. Floyd, and F.C. Cowlard: A comparative study of gaseous oxidation of vitreous carbon and various graphites at 1500-3000 degrees K. Carbon 6(2), 223 (1968).
6. J. Miklos, K. Mund, and W. Naschwitz: Patent DE 3011701 A1. Siemens AG and German Patent Office (1980).

7. A. Braun, M. Bärtsch, B. Schnyder, R. Kötz, O. Haas, H-G. Haubold, and G. Goerigk: X-ray scattering and adsorption studies of thermally oxidized glassy carbon. J. Non-Cryst. Solids 260(1-2), 1 (1999).

8. A. Braun, M. Bärtsch, F. Geiger, O. Haas, R. Kötz, B. Schnyder, M. Carlen, T. Christen, C. Ohler, P. Unternährer, H. Desilvestro, and E. Krause: A study on oxidized glassy carbon sheets for bipolar supercapacitor electrodes, in New Materials for Batteries and Fuel, edited by D.H. Doughty, L.F. Nazar, M. Arakawa, H-P. Brack, and K. Naoi (Mater. Res. Soc. Symp. Proc. 575, Warrendale, PA, 1999), p. 369.

9. A. Braun, M. Bärtsch, O. Merlo, B. Schaffner, B. Schnyder, R. Kötz, O. Haas, and A. Wokaun: Evolution of electrochemical double layer capacitance in glassy carbon during thermal oxidation-Crossover from exponential to logistic growth. Carbon 41(4), 759 (2003).

10. A. Braun, M. Bärtsch, B. Schnyder, R. Kötz, O. Haas, and A. Wokaun: Evolution of BET internal surface area in glassy carbon powder during thermal oxidation. Carbon 40(3), 375 (2002).

11. M. Bärtsch, A. Braun, B. Schnyder, R. Kötz, and O. Haas: Bipolar glassy carbon electrochemical double-layer capacitor: 100,000 cycles demonstrated. J. New Mater. Electrochem. Syst. 2, 273 (1999).

12. B.E. Conway and W.G. Pell: Double-layer and pseudocapacitance types of electrochemical capacitors and their applications to the development of hybrid devices. J. Solid State Electrochem. 7(9), 637 (2003)

13. A. Braun, J. Kohlbrecher, M. Bärtsch, B. Schnyder, R. Kötz, O. Haas, and A. Wokaun: Small angle neutron scattering and cyclic voltammetry study on electrochemically oxidized and reduced pyrolytic carbon. Electrochim. Acta 49(7), 1105 (2004).

14. W.S. Rothwell: Small-angle x-ray scattering from glassy carbon. J. Appl. Phys. 39(3), 1840 (1968).

15. J. Ilavsky, P.R. Jemian, A.J. Allen, F. Zhang, L.E. Levine, and G.G. Long: Ultra-small-angle x-ray scattering at the advanced photon source. J. Appl. Crystallogr. 42(3), 469 (2009).

16. J. Ilavsky and P.R. Jemian Irena: Tool suite for modeling and analysis of small-angle scattering. J. Appl. Crystallogr. 42(2), 347 (2009).

17. O. Glatter and O. Kratky: Small Angle X-Ray Scattering (Academic Press, New York, 1982).

18. M.E. Spahr, T. Palladino, H. Wilhelm, A. Würsig, D. Goers, H. Buqa, M. Holzapfel, and P. Novak: J Electrochem Soc. 151 (9), A1383 (2004)

19. H.J. Vogel: Morphological determination of pore connectivity as a function of pore size using serial sections. Eur. J. Soil Sci. 48(3), 365 (1997).

20. P. Wong and A.J. Bray: Scattering by rough surfaces. Phys. Rev. B 37, 7751 (1988).

21. A.J. Allen, J. Ilavsky, and A. Braun: Multi-scale microstructure characterization of solid oxide fuel cell assemblies with ultra small-angle x-ray scattering. Adv. Eng. Mater. 11(6), 495 (2009). 